\title{
Resistencias cotidianas a las políticas de rendición de cuentas o accountability: un estudio de casos de dos educadoras diferenciales en Chile
}

\author{
Daily Resistance to Accountability Policies: A Case Study of Two Special \\ Education Teachers in Chile
}

\section{Resistência diária às políticas de prestação de contas: um estudo de caso de dois educadores diferenciais no Chile}

\author{
Fabián Inostroza \\ Universidad de Las Américas, Facultad de Educación, Chile \\ fabian.uah.doc@gmail.com \\ http://orcid.org/0000-0003-1530-2266
}

\section{Resumen}

En Chile, la inclusión escolar es un ideal que se encuentra en jaque. En las últimas décadas, las políticas de rendición de cuentas o accountability se han legitimado como el discurso predominante en las escuelas. Estas instituciones escolares han priorizado la estandarización de los procesos educativos y la demostración de resultados por medio de evaluaciones como el Sistema de Medición de la Calidad de la Educación (Simce). La presente investigación, desde una perspectiva analítica foucaultiana, busca conocer las tácticas de resistencia que despliegan las educadoras diferenciales, actores encargados de la implementación de la inclusión, ante los mandatos de rendición de cuentas en la cotidianeidad. Por medio de un estudio de casos con enfoque etnográfico, en el que se realizaron entrevistas y observaciones participantes a dos educadoras diferenciales, se logra dar cuenta de la producción de tácticas colectivas e individuales para resistir a los procesos de estandarización de la enseñanza y de 


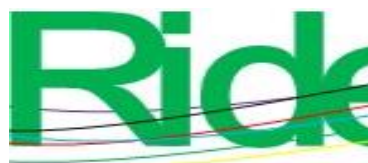

Revista Iberoamericana para la Investigación y el Desarrollo Educativo ISSN $2007-7467$

derivación de estudiantes con dificultades de aprendizaje a escuelas de educación especial, prácticas que resultan ser contrarias a la identidad y ética de estas docentes.

Palabras claves: educadoras diferenciales, inclusión escolar, políticas de rendición de cuentas, resistencia.

\section{Abstract}

In Chile, school inclusion is an ideal that is in check. In recent decades, accountability policies have been legitimized as the predominant discourse in schools. These institutions have prioritized the standardization of educational processes and the demonstration of results through assessments such as the System of Measurement of the Quality of Education (SIMCE). The present investigation, from a Foucauldian analytical perspective, seeks to know the resistance tactics displayed by the special education teachers, actors in charge of the implementation of inclusion, before the mandates of accountability in everyday life. Through a case study with an ethnographic approach, in which interviews and observations were conducted with two special education teachers, it is possible to realize the production of collective and individual tactics to resist the standardization processes of the teaching and referral of students with learning disabilities to special education schools, practices that turn out to be contrary to the identity and ethics of these teachers.

Keywords: special education teachers, school inclusion, accountability policies, resistance.

\section{Resumo}

No Chile, a inclusão escolar é um ideal que está em xeque. Nas últimas décadas, políticas de responsabilização ou responsabilização foram legitimadas como o discurso predominante nas escolas. Essas instituições escolares priorizaram a padronização dos processos educacionais e a demonstração de resultados por meio de avaliações como o Sistema de Medição da Qualidade Educacional (Simce). A presente investigação, na perspectiva analítica de Foucault, busca compreender as táticas de resistência empregadas por educadores diferenciais, atores encarregados da implementação da inclusão, diante dos mandatos de prestação de contas no cotidiano. Por meio de um estudo de caso com abordagem etnográfica, em que foram realizadas entrevistas e observações dos participantes com dois educadores diferenciais, é possível dar conta da produção de táticas coletivas e individuais para resistir 


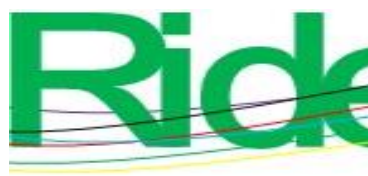

Revista Iberoamericana para la Investigación y el Desarrollo Educativo ISSN $2007-7467$

centro. Una política emblemática en este sentido es la Ley de Subvención Escolar Preferencial (SEP) $)^{2}$ (Assaél, Acuña, Contreras y Peralta, 2014; Fardella y Sisto, 2015).

En un escenario como el descrito, la rendición de cuentas y la demostración de resultados en la evaluación Simce $^{3}$ se transforman en la prioridad, dejando las otras iniciativas educativas, como la inclusión, como procesos de segundo orden, ya que las escuelas y los actores se encuentran tensionados y (pre)ocupados por el cumplimiento de los indicadores de calidad impuestos por las políticas.

Es en estas escuelas en donde un actor educativo en especial debe producir un trabajo que se torna contracultural respecto a la estandarización y la homogeneización del alumnado: las y los educadores diferenciales, profesionales de la educación encargados de la inclusión escolar en las escuelas chilenas, quienes desempeñan su trabajo por medio de un conjunto de acciones pedagógicas para apoyar a los estudiantes con dificultades de aprendizaje en general y de forma específica a los educandos que han sido categorizados con necesidades educativas especiales (NEE).

Al respecto, la literatura científica existente en Chile demuestra una escasa investigación empírica sobre estas docentes. Salvo una excepción, representada por el estudio de Inostroza (2020), las investigaciones se han encargado principalmente de examinar los desafíos que las políticas de inclusión escolar han significado para su identidad docente y desempeño profesional y no han prestado atención a los efectos de las políticas de rendición de cuentas en su trabajo pedagógico cotidiano.

En suma, en instituciones escolares que se han caracterizado históricamente por una gramática escolar focalizada en la homogeneización y normalización de educandos, aspecto que ha sido amplificado e intensificado por la estandarización de los procesos educativos que promueven la rendición de cuentas con altas consecuencias (Ball, 2016; Tyack y Cuban,

\footnotetext{
${ }^{2}$ La SEP otorgó un aumento de $60 \%$ de la subvención escolar regular por estudiante vulnerable condicionando la entrega de estos recursos a la elaboración de un PME, que es evaluado fundamentalmente por medio de los puntajes en la evaluación Simce en Lenguaje y Matemáticas en 4. ${ }^{\circ}$ grado de educación básica o primaria.

${ }^{3}$ El Simce corresponde a una evaluación estandarizada de carácter anual y censal que evalúa los rendimientos de los alumnos en asignaturas como Lenguaje, Matemáticas, Ciencias Sociales y Ciencias Naturales en los niveles de $4 .^{\circ}$ grado de primaria, $8 .^{\circ}$ de grado de primaria y $2 .^{\circ}$ año de educación secundaria. En virtud de los resultados en esta prueba, se evalúan los proyectos de mejoramiento de las escuelas, se categoriza a los centros escolares y se les premia o sanciona.
} 


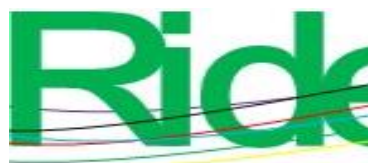

Revista Iberoamericana para la Investigación y el Desarrollo Educativo ISSN $2007-7467$

2001), desplegar discursos y prácticas tendientes a la inclusión escolar se torna un trabajo contracultural y altamente tensionado. Por tanto, la presente investigación pretende responder al siguiente interrogante: ¿cuáles son las tácticas de resistencia que despliegan dos educadoras diferenciales ante los mandatos de las políticas de rendición de cuentas en su trabajo pedagógico cotidiano?

\section{Marco teórico}

\section{Políticas de rendición de cuentas o accountability}

Las políticas de rendición de cuentas o accountability surgen en la década de los 80 del siglo pasado con la finalidad de reformar al sistema público: brindarle mayor dinamismo al lento y burocrático accionar del Estado, incorporando e importando modelos de gestión empresarial en la provisión de los servicios públicos, tales como la educación (Verger, Bonal y Zancajo, 2016).

Específicamente en el ámbito educativo, las políticas de rendición de cuentas pueden ser definidas en términos de una relación contractual que se establece entre un actor específico (escuela) y una contraparte, que usualmente corresponde al Estado. En este contrato, las escuelas y los actores escolares se encuentran obligados a rendir cuentas respecto de sus conductas y en cuanto al uso del financiamiento recibido por parte del Estado central o federal. En este sentido, el Estado como ente que financia a las instituciones educativas tiene el derecho a exigir evidencias, supervisar y producir consecuencias para los establecimientos educacionales que no cumplan con las metas educativas comprometidas en cuanto a calidad (Parcerisa y Falabella, 2017).

En cuanto a taxonomías del accountability educativo, a nivel internacional se destaca la clasificación elaborada por Darling-Hammond (2004), quien distingue cinco tipos: político, legal, burocrático, profesional y mercado. Mientras que Maroy y Voisin (2013) producen otra distinción entre modelos de accountability: strong accountability, de tipo neoburocrático, reflexivo y soft accountability. Para el caso de Chile, los investigadores Falabella y de la Vega (2016), a través de una revisión de literatura, proponen que, en la actualidad a nivel mundial, los enfoques de rendición de cuentas más utilizados corresponden al estatal o burocrático, por desempeño o de mercado y el profesional. 


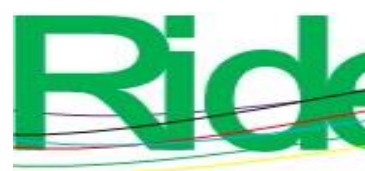

Revista Iberoamericana para la Investigación y el Desarrollo Educativo ISSN $2007-7467$

En el caso del sistema educativo chileno, el enfoque o tipo de accountability que ha guiado las últimas políticas educativas (por ejemplo, la SEP) es el denominado por la literatura como de responsabilización por desempeño o de mercado (Falabella y de la Vega, 2016; Parcerisa y Falabella, 2017). Este tipo de rendición de cuentas se caracteriza por una relación entre el Estado y las escuelas que reciben el financiamiento, en donde las instituciones educativas se comprometen a mejorar la calidad de la educación que ofrecen a sus educandos, calidad que es medida en un porcentaje relevante por medio de la evaluación estandarizada Simce. En caso que la institución escolar no mejore sus desempeños, en un plazo determinado (cuatro años aproximadamente) esta puede perder consecuentemente el reconocimiento oficial y ser eventualmente cerrada.

\section{Poder y resistencia}

La noción de resistencia será comprendida en esta investigación desde un enfoque foucaultiano, perspectiva en la que no se puede hablar de resistencia sin antes hacer alusión al poder. El poder desde Foucault no responde a una capacidad adquirida o heredada que se ejerce de manera unidireccional, sino que más bien corresponde a una fuerza omnipresente y constitutiva de las relaciones humanas; un poder que es ejercido en diversas direcciones y que al mismo tiempo es un objeto de lucha (Foucault, 2013). Por tanto, para el pensador francés en tanto exista una relación de poder en la interacción entre los sujetos, existirá la posibilidad de resistencia a este.

Lo novedoso de esta noción de resistencia es que se distingue de concepciones más tradicionales en donde es representada como la imagen invertida del poder. En este sentido, más que una fuerza antagónica que se ejerce unidireccionalmente por los sujetos para escapar a la dominación por parte de una gran estructura que oprime, para Foucault (1994) la resistencia es "tan inventiva, tan móvil, tan productiva como el poder. Por ello es que es preciso que se organice, se coagule y se cimiente. Que vaya de abajo hacia arriba, y como el poder, se distribuya estratégicamente" (p. 114).

Es decir, desde la analítica foucaultiana, la resistencia corresponde al despliegue de estrategias y de tácticas que permiten a los sujetos contrarrestar la sujeción de determinados poderes, comprendida esta relación como fuerzas que se encuentran y colisionan en diversos puntos en el marco de las relaciones humanas. En este sentido, la resistencia puede ser producida como una contraconducta, como una forma de fisura o una línea de fuga en la que 


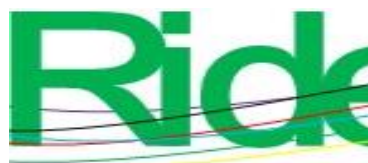

Revista Iberoamericana para la Investigación y el Desarrollo Educativo ISSN $2007-7467$

los sujetos pueden cambiar y modificar de manera creativa y transformadora la distribución del poder a su favor (Castro, 2004; Foucault, 2013).

En consecuencia, y retomando el trabajo del pensador francés respecto al gobierno de las subjetividades, Foucault (2013) propone que desde el Estado se busca gobernar o conducir las conductas de los sujetos, limitando el campo de actuaciones posibles a un número restringido de acciones o de formas de construir el yo (self) para la población. En esta misma línea de argumentación es que conceptualiza lo que correspondería a una contraconducta, la que puede ser definida como toda acción que un sujeto consciente despliega y que escapa o se distancia de las conductas esperadas y deseadas desde la mentalidad de gobierno o la gubernamentalidad en este espacio-tiempo (Foucault, 2006).

A modo de ejemplo, cada vez que un sujeto docente despliega tácticas concertadas, individuales o colectivas que sean "no esperadas o no deseadas" por los discursos de las políticas y que, a su vez, escapen a la norma de lo que corresponde en esta sociedad y en este espacio-tiempo a lo que "un buen educador" debería hacer o ser, se estaría en presencia de una resistencia, la que se materializaría por medio de un haz o conjunto de contraconductas (Castro, 2004).

\section{Marco metodológico}

\section{Diseño}

Este estudio responde a una investigación de tradición cualitativa que considera como enfoque epistémico a la perspectiva poscrítica de investigación social (Da Silva, 2001; Larraín, 2011).

Esta investigación se realizó contemplando dos fases. En una primera etapa se llevaron a cabo entrevistas en profundidad a 10 educadoras diferenciales de diversos establecimientos educacionales de la Región Metropolitana de Chile, quienes proporcionaron sus discursos respecto a la inclusión escolar, las políticas de rendición de cuentas y las políticas educativas en general. De estas docentes, se seleccionó a dos pedagogas, quienes manifestaron diversas perspectivas respecto a la rendición de cuentas y que, a su vez, se desempeñaban en escuelas que se encontraban en posiciones diametralmente opuestas en cuanto a la demostración de desempeños según la evaluación estandarizada Simce: una institución que estaba clasificada como "en recuperación" y otra que estaba como 


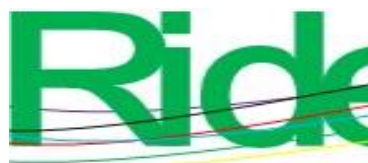

\section{Revista Iberoamericana para la Investigación y el Desarrollo Educativo ISSN 2007 - 7467}

“autónoma”. Es preciso señalar, además, que el trabajo de campo de este estudio se realizó durante el año 2018.

En términos generales, una escuela clasificada "en recuperación" es aquella que ha demostrado bajos rendimientos a nivel de la evaluación Simce en $4 .^{\circ}$ año de primaria en Lenguaje y Matemáticas, y que, en el caso de no mejorar sus resultados, se le revoca el reconocimiento oficial y corre el riesgo de ser cerrada. En tanto, un centro escolar categorizado como "autónomo" es aquel que ha demostrado resultados altos en el Simce a nivel de $4 .^{\circ}$ grado de primaria y, por consecuencia, tiene un menor grado de supervisión y de presión por mejorar su desempeño en la evaluación estandarizada ya mencionada.

Esta investigación en particular se centró en la segunda etapa, en el seguimiento etnográfico de estas dos educadoras, con la finalidad de profundizar en sus discursos, así como para evidenciar en sus contextos de desempeño el cómo desplegaban resistencia por medio de contraconductas ante las lógicas de rendición de cuentas instaladas en las escuelas a través de la SEP.

En cuanto al diseño, este responde a un estudio de caso con enfoque etnográfico (Guber, 2004; Rockwell, 2009). Como ya se mencionó, se realizó una selección de participantes por medio de un muestreo cualitativo e intencionado: se escogieron a dos educadoras diferenciales que se desempeñaban en establecimientos educacionales subvencionados por el Estado de Chile y que se encontraban adscritas a las políticas de rendición de cuentas o accountability. Anteriormente, en el caso de las primeras 10 participantes, se escogió el mismo número de escuelas y se les envió una carta de invitación para que alguna de las educadoras que en estos centros se desempeñaban pudiesen participar y luego firmar los correspondientes consentimientos informados. Posteriormente, las dos docentes que fueron parte de los casos de estudio fueron escogidas por medio de tres criterios fundamentales para esta investigación: 1) el grado de afinidad con las políticas de rendición de cuentas, 2) la clasificación de la escuela de acuerdo con su rendimiento en el Simce y 3) el nivel educativo en el que ejercían (primer o segundo ciclo de educación primaria). 


\section{Revista Iberoamericana para la Investigación y el Desarrollo Educativo ISSN $2007-7467$}

\section{Descripción de las participantes}

Una de las participantes denominada en este estudio como Claudia ${ }^{4}$ se desempeñaba en una de una escuela municipal de alta vulnerabilidad social (98\%), institución que se encontraba "en recuperación" de acuerdo con la clasificación producida por la Agencia de Calidad de la Educación (2019). Y la otra educadora, aquí denominada Pamela, pertenecía a un colegio particular subvencionado, institución que era administrada por una congregación religiosa católica, que contaba con un nivel de vulnerabilidad menor (alrededor de $80 \%$ ) y que estaba categorizada como "autónoma" de acuerdo con datos aportados por la Agencia de la Calidad de la Educación (2019).

En la tabla 1 se resumen las características de las participantes correspondientes a los casos de estudio.

Tabla 1. Descripción de los casos de estudio

\begin{tabular}{|c|c|c|}
\hline Características de las educadoras & Claudia & Pamela \\
\hline $\begin{array}{l}\text { Afinidad con las políticas de } \\
\text { accountability }\end{array}$ & Menor grado & Mayor grado \\
\hline Edad (años) & 32 & 36 \\
\hline Años de trabajo en el establecimiento & 7 & 10 \\
\hline Cargo & $\begin{array}{l}\text { Segundo ciclo (de } 5 .^{\circ} \text { a } \\
8 .^{\circ} \text { grado de primaria) }\end{array}$ & $\begin{array}{l}\text { Primer ciclo (de } 1 .^{\circ} \text { a } \\
4 .^{\circ} \text { grado de primaria) }\end{array}$ \\
\hline \multicolumn{3}{|l|}{ Características de la institución } \\
\hline Dependencia & Municipal (público) & $\begin{array}{c}\text { Particular } \\
\text { subvencionado }\end{array}$ \\
\hline Nivel de vulnerabilidad del alumnado ${ }^{5}$ & $98 \%$ & $80 \%$ \\
\hline Clasificación SEP de la escuela & En recuperación & Autónoma \\
\hline
\end{tabular}

Fuente: Elaboración propia

\footnotetext{
${ }^{4}$ Se han denominado a los casos de estudio de esta manera para resguardar su identidad y respetar el acuerdo de confidencialidad presente en los consentimientos informados.

${ }^{5}$ El índice de vulnerabilidad escolar (IVE) es un indicador elaborado por la Junta Nacional de Auxilio Escolar y Becas (Junaeb) para medir el grado de riesgo social y pobreza de los educandos del sistema escolar chileno.
} 


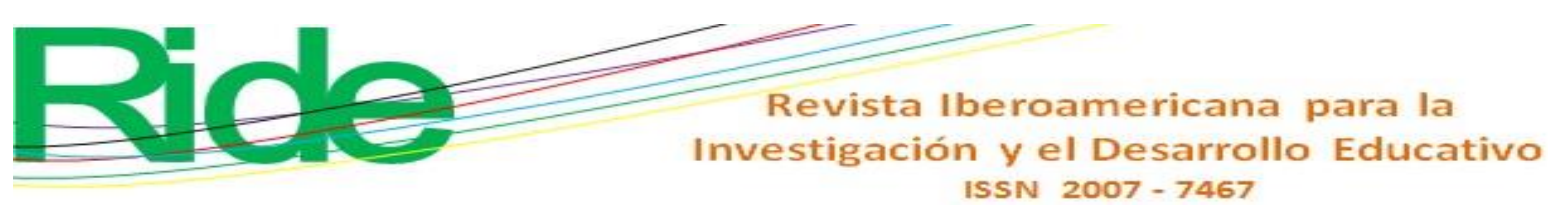

\section{Procedimientos}

En cuanto a los procedimientos, a cada una de estas dos participantes se le realizó un seguimiento etnográfico durante un semestre académico (lo que equivalió a cinco meses por caso de estudio), periodo en el que se desarrolló el trabajo de campo. Durante este proceso se les aplicó a las educadoras entrevistas en profundidad (dos a cada una), observación no participante (una vez por semana durante una jornada laboral) y se les solicitó escribir una bitácora reflexiva a cada una de ellas. Dicha bitácora consistía en describir, empleando sus propias palabras, en un documento con una extensión de no más de dos páginas, una jornada de trabajo pedagógico "típica", de tal forma que se pudiera contar con un registro escrito en el que estuviera presente la experiencia vivida de las participantes en sus rutinas laborales, y con ello lograr captar con mayor riqueza y profundidad la influencia de las políticas en su quehacer diario.

En total, el corpus discursivo recopilado durante el trabajo de campo contó con cuatro entrevistas en profundidad (equivalentes a seis horas de grabación), 120 notas de observación y 10 bitácoras producidas por las propias educadoras.

\section{Técnicas de análisis de la información}

En términos de la técnica de análisis del corpus discursivo producido en esta investigación, este proceso de dividió en dos fases. En una primera instancia, este se orientó por medio del análisis de contenido (Bardin, 1996), en el que se empleó de forma instrumental el software de análisis cualitativo Atlas Ti versión 7.1 para desarrollar una sistematización y organización preliminar del material cualitativo.

Una vez desarrollada una primera sistematización de la información, de la que emergieron categorías preliminares, se procedió, en una segunda etapa, a realizar un análisis con enfoque discursivo orientado por las claves teóricas-analíticas aportadas por Foucault (2013). Es decir, se analizaron las resistencias a las políticas en virtud de las contraconductas que produjeron las educadoras en sus contextos de desempeño institucional en su trabajo pedagógico cotidiano.

Específicamente, se elaboró una matriz de análisis de doble entrada en la que se dispuso de columnas con los respectivos instrumentos de recogida de información desde donde provenían los discursos seleccionados: entrevista, notas de campo y bitácoras. En tanto 


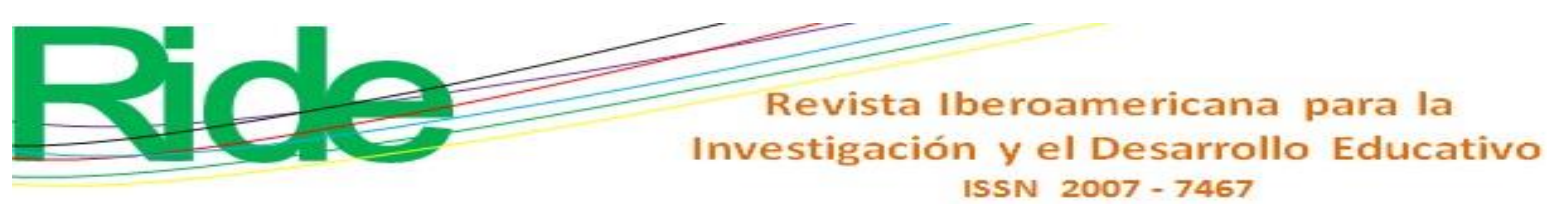

que en las filas se consignó a las categorías teóricas provenientes de la analítica foucaultiana, tal y como las relaciones de poder presentes materializadas por medio de las resistencias ante la estandarización y ante la derivación de alumnos a escuelas de educación especial.

\section{Resultados}

Entre los hallazgos más relevantes que emergieron del proceso de análisis del corpus discursivo se encuentra una resistencia por parte de las educadoras a los procesos de estandarización de la enseñanza y ante el mandato de derivación de alumnos con dificultades de aprendizaje a escuelas de educación especial. Estas contraconductas que las participantes desplegaron se dieron tanto de forma individual como colectiva.

\section{Resistencias ante la estandarización: entrenamiento del alumnado para la evaluación Simce}

En cuanto a las prácticas de estandarización, se constataron en los discursos y episodios diversas y creativas formas de resistencia por parte de las educadoras. Esta desobediencia se produjo de diferentes maneras en los casos de estudio. Cabe recordar que, en el marco de esta investigación, se denominará a las participantes como Claudia y Pamela 6 . Por un lado, las contraconductas que Claudia desplegó fueron limitadas: dado el contexto educativo en el que se desempeñaba, estaba altamente presionada por obtener "buenos" resultados en el Simce. Por el otro, en Pamela la desobediencia fue manifestada de manera más explícita ante los mandatos de trabajar en favor de la evaluación estandarizada.

A continuación, se exponen discursos y episodios en los que las participantes desplegaron resistencia a las lógicas de la estandarización. Primeramente se revela el caso de Pamela, quien expuso de esta manera su posición respecto al proceso de entrenamiento de alumnos para el Simce:

\footnotetext{
${ }^{6}$ También es importante recordar que Claudia es la educadora que se desempeñaba en la escuela municipal con alto nivel de vulnerabilidad, institución que se encontraba clasificada como "en recuperación". Mientras que Pamela es la docente que trabajaba en la escuela de dependencia particular subvencionada, con un nivel de vulnerabilidad del alumnado menor y que estaba categorizada como "autónoma" o con "desempeño medioalto".
} 


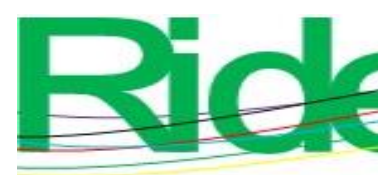

Revista Iberoamericana para la
Investigación y el Desarrollo Educativo
ISSN $2007-7467$

Bueno, los meses antes del Simce son bien complicados, agosto, septiembre y octubre. Te desordenan tu jornada cuando te mandan a trabajar con los niños del PIE para el Simce. Pero a ver, igual uno puede hacer trampa, como se dice, con el tema del Simce. A veces me han mandado a trabajar con los terceros y cuartos para apoyarlos con el tema Simce, pero yo me los traigo al aula de recursos y acá trabajo en la nivelación de sus habilidades matemáticas o de lenguaje, pero hacemos el trabajo diferenciado que yo decida, acá nadie me vigila. Al final, igual en el libro de clases puedo poner apoyo a profesora de aula, entonces depende de uno seguir la corriente o ir en contra (Pamela, 7 de septiembre de 2018, entrevista).

En este fragmento, la educadora manifiesta la posibilidad de resistir a los mandatos de la política de rendición de cuentas, los cuales se cristalizan por medio de las órdenes emanadas de la dirección del colegio en el que se desempeñaba. La participante pudo producir estas "trampas" o contraconductas en un espacio en donde no es vigilada u observada de manera directa, a saber, el aula de recursos. ${ }^{7}$ De hecho, este lugar es uno de los sitios al interior de las escuelas en el que la investigación empírica no ha prestado la atención suficiente para adentrarse en la compresión de las resistencias que emergen a raíz de las demandas de estandarización de los procesos educativos.

Es precisamente en este espacio donde Pamela mencionó que puede "hacer trampa", es decir, esta docente tiene la posibilidad de ejercer un trabajo diferenciado acorde a su criterio profesional como educadora, desobedeciendo a los mandatos de preparar explícitamente a los educandos con dificultades de aprendizaje para rendir en la evaluación Simce.

Este hallazgo es concordante con episodios observados en las instituciones educativas en las que se desempeñaban las participantes, ya que se registró que el aula de recursos se constituyó en un espacio en el que ambas docentes desobedecieron los discursos de estandarización promovidos por las políticas de rendición de cuentas, en específico la ley SEP, la cual las interpelaba para trabajar en obtener "altos" puntajes en la evaluación Simce.

\footnotetext{
${ }^{7}$ El aula de recursos corresponde el espacio pedagógico al interior de los PIE, en donde las educadoras desarrollan un trabajo personalizado y ajustado a las necesidades educativas de los educandos categorizados con dificultades del aprendizaje.
} 


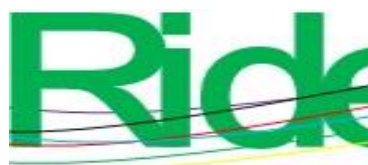

\section{Revista Iberoamericana para la Investigación y el Desarrollo Educativo ISSN 2007 - 7467}

A modo de ejemplo, esta resistencia fue expresada por medio de acuerdos colectivos en el caso de Claudia, a través de la producción de una aparente "obediencia" ante el mandato de entrenar a los estudiantes para el Simce.

En el contexto de una escuela que se encontraba altamente tensionada y con un estrés desbordado ante la amenaza de cierre, en donde los actores educativos dirigían sus conductas hacia la consecución de un "buen puntaje" en el Simce, las educadoras no contaban con la posibilidad de desobedecer explícitamente, pero sí produjeron una táctica colectiva de resistencia en el aula de recursos para aplicar a los educandos los ensayos para la evaluación estandarizada con un porcentaje significativamente menor de preguntas y con un mayor enfoque en atender las dificultades de aprendizaje del alumnado.

Coordinador del PIE: Ya, muchachas, tenemos que decidir cómo nos vamos a organizar para el tema de los cuartos básicos.

Claudia: De verdad, no estoy de acuerdo, pero hay que ir a la sala y apoyar, no queda otra. Ahora, para qué les vamos aplicar el ensayo completo o tal cual a los niños del PIE, con tal que hagan unas 10 preguntas y basta, lo importante es aprovechar de repasar los contenidos que están viendo el curso, si no nos pagan para calentar el Simce.

Educadora 2: Estoy de acuerdo, como están las cosas en la escuela hay que apoyar, pero hay que tomarlo como una forma de reforzar contenidos con los niños del PIE, y no importa si hacemos 5 o 10 preguntas, o por último una pregunta, lo importante es apoyarlos en las habilidades más descendidas y el resto del Simce no me importa.

Coordinador del PIE: Bien, que se note que es una decisión que apoyamos, que se den cuenta de dirección que vamos a los cuartos básicos y que estamos trabajando con los niños del PIE. Más allá, no nos van a supervisar (20 de junio de 2018, nota de campo).

En este episodio se puede observar que se llegó a un acuerdo colectivo por parte del coordinador y las educadoras diferenciales para resistir a los mandatos de estandarización, aplicando un criterio profesional enfocado en trabajar en el aula regular con los educandos con dificultades del aprendizaje. De esta forma, se estaba cumpliendo con lo solicitado por la dirección y al mismo tiempo desplegando contraconductas vinculadas a su función como encargadas de la inclusión al interior de la escuela. Es así que, en este contexto educativo, se 


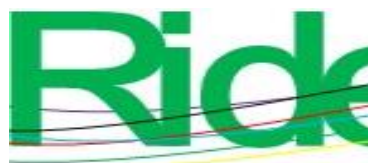

Revista Iberoamericana para la
Investigación y el Desarrollo Educativo
ISSN $2007-7467$

estaría desobedeciendo de una manera más "velada" a la normalización, en términos foucaultianos, dirigida a la elaboración de subjetividades de estudiantes productivos en función del Simce.

Mientras que en el caso de Pamela, en el escenario de la aplicación de ensayos previos a que los alumnos rindieran el Simce, de manera excepcional se le permitía a esta docente retirar al alumnado con dificultades de aprendizaje de este colegio y realizar estas evaluaciones en el aula de recursos. En este episodio se manifestó la siguiente forma en que la educadora "desobedeció" a la práctica a la que era interpelada:

(La educadora ya fue a buscar a los niños de cuarto básico a la sala de clases y les indicó que se sentaran en la mesa grande del aula de recursos).

Pamela (dirigiéndose a los niños): Ya, niños, nos toca trabajar con estas guías del Simce, pero ¿recuerdan que la semana pasada en matemáticas estábamos trabajando con las fracciones y ocupamos un material que se llama regletas de Cuisenaire?

Niños: Sí (de manera coral).

Pamela (guarda los ensayos Simce en su escritorio): Bien, entonces, vamos a seguir trabajando con eso.

Educadora Carmen: Como son seis, trabajarán en parejas y les pasaré las regletas y una guía que les tenía preparada. Eso es lo que haremos hoy (26 de septiembre de 2018, nota de campo).

En este episodio queda en evidencia que Pamela desobedeció a los mandatos de realizar un ensayo de preparación para la prueba Simce, desacato que materializa llevándose a los alumnos con dificultades de aprendizaje del cuarto básico (o cuarto año de primaria) al aula de recursos. Similar a lo ocurrido en el caso de Claudia, la educadora Pamela desarrolló un trabajo pedagógico alternativo a los ensayos para el Simce, actividad pedagógica que si bien correspondía a la misma asignatura (matemáticas) no tenía una relación directa con responder un instrumento de evaluación estandarizado que emulaba al Simce. Por tanto, en el caso de Pamela, se detectó una táctica de resistencia individual en el aula de recursos.

En estos dos episodios presentados con anterioridad se ven reflejadas diversas formas en que las participantes Claudia y Pamela, respectivamente, resistieron a las lógicas que instalaban las políticas de rendición de cuentas por medio del despliegue de contraconductas, si bien obedecían (en apariencia) a los mandatos de las respectivas instituciones escolares. 


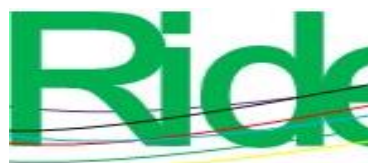

Revista Iberoamericana para la Investigación y el Desarrollo Educativo ISSN $2007-7467$

Asimismo, es en el aula de recursos en donde ellas deliberaron y ejercieron conductas que están vinculadas a su criterio profesional como educadoras diferenciales, las que pueden ser conceptualizadas como resistencia a los discursos que promovía el accountability en sus respectivos contextos de desempeño profesional.

\section{Resistencia ante la derivación de alumnos a escuelas de educación especial}

Dentro de las prácticas que produjeron las participantes y que las mismas denominaron como "de exclusión” y que, por lo demás, fueron constatadas con mayor frecuencia en el caso de Claudia y con una menor intensidad para la educadora Pamela, fue la derivación de educandos con mayores dificultades en el aprendizaje en las asignaturas de Lenguaje y Matemáticas a escuelas de educación especial.

Estas prácticas, de acuerdo con el criterio profesional de las docentes pertenecientes a los casos de estudios, correspondían a la exclusión de alumnos que ellas como educadoras diferenciales o sus colegas encargadas de la inclusión paradójicamente tuvieron que asumir, dado el mandato de las respectivas instituciones educativas en las que se desempeñaban, acciones que a juicio de estas pedagogas iban en contra de toda ética profesional.

Particularmente, tanto en los discursos como en los episodios observados, se detectaron en los casos de Claudia y Pamela esta exclusión de alumnos a escuelas de educación especial, la que fue mencionada en uno de los casos como un mandato expreso (Claudia). En cambio, Pamela sugirió en su discurso que este tipo de prácticas no se daban en el colegio en el que se desempeñaba, lo que no se condice con las conductas que fueron identificadas en la institución educativa en la que se desenvolvía profesionalmente.

Al ser consultadas estas educadoras respecto a la existencia de este tipo de prácticas en los centros escolares en los que se desempeñaban, estas docentes se refirieron de la siguiente forma a esta temática. Primero Claudia:

Es un tema delicado, pero no puedo negar que aquí se ha hecho. El tema no se habla mucho, porque es contrario a la inclusión y para mí no debería pasar en ninguna circunstancia, pero, como lo hablábamos antes, el tema del Simce y de que la escuela esté peligrando produce este tipo de cosas. A una colega le tocó derivar a una niña a una escuela especial y todos sabíamos que esa niña no tenía nada tan serio como para mandarla a una escuela especial, pero aquí nos presionan de todos lados y pasó. Pero, como te digo, no se habla el tema, 


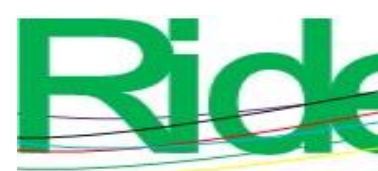

Revista Iberoamericana para la
Investigación y el Desarrollo Educativo
ISSN $2007-7467$

porque en su momento fue muy fuerte y lo sigue siendo (Claudia, 5 de junio de 2018, entrevista).

Pamela, por su parte, mencionó lo siguiente:

Bueno, yo estoy en conocimiento de que es algo que pasa y no digo que no exista, porque he escuchado de boca de otras colegas de otros colegios que lo hacen, pero en este colegio no se ha hecho y yo llevo hartos años trabajando aquí y me hubiera enterado. Yo sé que para algunas personas los niños con dificultades son un problema y que no rinden bien en el Simce, pero no es razón para derivarlos. Pero sé que en otras escuelas es una práctica bien común, como esconder a los niños con dificultades el día del Simce, por decirte (Pamela, 10 de noviembre de 2018, entrevista).

En concordancia con lo señalado, en los contextos escolares en los que se desempeñaban Claudia y Pamela también se evidenció que, en la práctica, estas docentes desplegaron resistencia ante los mandatos de excluir a estudiantes con dificultades de aprendizaje, aunque esta desobediencia emergió principalmente en el caso de Claudia, quien, empleando el aula de recursos, citó estratégicamente a una apoderada de una alumna que estaba siendo evaluada para ser eventualmente derivada a una institución de educación especial dados sus bajos rendimientos en asignaturas claves para el currículo chileno y para la evaluación Simce, como lo son Lenguaje y Matemáticas. De esta forma se dio la reunión entre Claudia y la apoderada de la alumna que, desde la escuela regular, se pretendía derivar:

Claudia: Señora Juanita, tenga en cuenta que este año a la Jacinta le ha ido muy mal y eso nos preocupa, porque de no mejorar y terminar con promedio rojo este semestre habría que volver a evaluarla y en una de esas enviarla a otra escuela, aunque no es lo que yo quiero para ella, por eso la llamaba.

Apoderada: Pero no le entiendo, señorita, ¿cómo llevarla a otra escuela?

Claudia: Es que la escuela tiene una postura bien estricta porque están con bajos resultados y los niños que no rinden bien los mandan a escuelas especiales.

Apoderada: ¡Ah, no! No, yo no quiero que a mi hija la saquen de aquí y la manden a ese tipo de escuelas, porque ahí van solo niños con muchos problemas y mi niña es normal y solo le cuesta un poco más. 


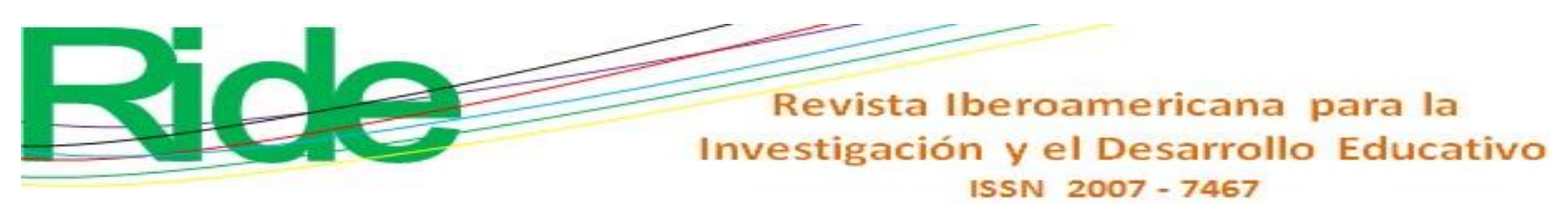

Claudia: Por eso la cité, para que podamos trabajar juntas y usted la pueda apoyar desde la casa, para que mejore sus notas y permanezca en el colegio y así no la mandan a la escuela especial (7 de mayo de 2018, nota de campo).

En el episodio presentado, la educadora Claudia, ante el mandato de la dirección, se anticipó y solicitó colaboración de la apoderada de la alumna a la que se buscaba derivar, de forma tal que la estudiante en cuestión no fuese excluida de la escuela regular. Esta situación se da ante la contingencia del centro escolar en el que desempeñaba Claudia, el cual, recordemos, estaba clasificado "en recuperación" y que, por tanto, se encontraba ante la urgencia de demostrar un aumento significativo en los puntajes de la evaluación Simce, y así evitar perder el reconocimiento oficial y no tener que ser cerrada.

Ante la contingencia mencionada, se les indicó desde dirección de esta escuela a las educadoras que evaluaran a los niños con más bajo rendimiento en $4 .^{\circ}$ año básico (primaria) en las asignaturas de Lenguaje y Matemáticas. Claudia, consciente de que este tipo de exclusión iba en contra de su criterio profesional, presentó una contraconducta "velada" desde el rango restringido de acciones que podía desplegar, ya que esta pedagoga también dependía de la dirección del establecimiento para mantener su empleo, debido a que su régimen laboral no estaba asociado a un contrato indefinido, sino que a uno de renovación anual, por medio de honorarios, recursos que eran financiados a través de la SEP.

La posibilidad de resistencia y de producción de contraconductas de las participantes dependía de ciertos factores que fueron pesquisados. En el caso de Claudia y Pamela, se evidenció que las presiones laborales y las condiciones contractuales en las que se encontraban influían en el ejercicio de resistencia que podían crear ante las prácticas de derivación de alumnos con dificultades del aprendizaje a escuelas de educación especial.

A propósito de las limitaciones contractuales, se presenta un episodio en el que Pamela, al dialogar con una de sus colegas del colegio, comenta la situación de una docente que se opuso de manera explícita a la derivación de alumnos a una escuela especial, lo que implicó que esta pedagoga fuera desvinculada (despedida) de su trabajo.

Pamela: ¿Supiste lo de la María? La fueron hace poco.

Educadora Rosa: Sí po. Estaba cantado. Pero era decisión de ella en todo caso. Nosotros la aconsejamos, pero lo bueno es que ella es joven, sin 


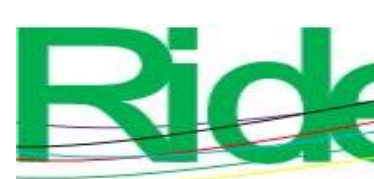

Revista Iberoamericana para la
Investigación y el Desarrollo Educativo
ISSN $2007-7467$

familia ni hijos. En mi caso, no me hubiese negado a derivar al niño, porque tengo cuentas que pagar y familia.

Pamela: Lamentablemente. Deberíamos estar de planta y tener trabajo indefinido y así, si te obligan a hacer una derivación, uno poder negarse nomás y en caso de querer echarte, uno va pa inspección. Pero ahora, depende de uno, por más que uno necesite plata, si me hubiesen obligado derivar, no lo hubiera hecho, siempre la ética primero.

Educadora Rosa: Eso lo dices porque igual llevas más años que las demás en la escuela y te consideran. En cambio, una, como la María, si te niegas, te echan nomás y no hay a quien reclamar (15 de mayo de 2018, nota de campo).

En el diálogo presentado, es posible identificar que existían factores en esta escuela que dificultaban el ejercicio de resistencia por parte de estas dos educadoras ante una eventual petición por parte de la dirección tendiente a la derivación de algunos alumnos con dificultades de aprendizaje a una institución de educación especial. Estos factores o variables estaban vinculadas con las precarias e inestables condiciones laborales y contractuales bajo las que se encontraban estas profesionales de la educación en la institución escolar en cuestión.

En el episodio presentado, Pamela fue enfática al señalar que, a pesar de estar amenazado su trabajo en el colegio, en el caso de que se le solicitara derivar a un alumno a una escuela de educación especial, es su ética la que debe prevalecer en su rol de educadora diferencial y, por tanto, preferiría perder su trabajo a obedecer al mandato de derivación. Además, otro aspecto a relevar en su discurso es que Pamela reconoció de manera explícita que, en esta institución educativa, la práctica de exclusión de educandos "sí sucedía o había sucedido", lo que resulta contrario a lo que afirmó esta docente en la primera entrevista en profundidad que ofreció en el marco de esta investigación.

Aunado a estos episodios y relatos, fue en las bitácoras reflexivas en las que tanto Claudia como Pamela manifestaron su tensión ante estas dinámicas de estandarización/exclusión en las que fueron hasta cierto punto actores partícipes, al estar inmersas en contextos educativos tensionados (en diversos grados) por la rendición de cuentas con altas consecuencias (high stakes accountability). 


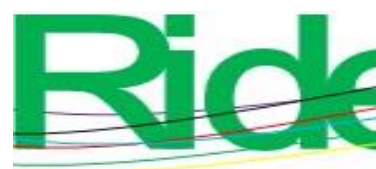

Revista Iberoamericana para la Investigación y el Desarrollo Educativo ISSN $2007-7467$

En este sentido, Claudia construyó una narrativa de su labor diaria en la que retrató esta tensión en su trabajo: la de un actor que incluye y al mismo tiempo contribuye a la exclusión en la escuela en la que se desempeñaba. De esta forma lo expuso en una de las bitácoras que produjo en el marco de este estudio:

Entonces, siempre cuando termina un día, me voy para la casa pensado: ¿es lo que yo quiero como diferencial? ¿Que se excluya a todos los estudiantes que no rinden bien en el Simce o que son problemáticos para los profes y para el colegio? ¿En eso se ha convertido nuestra pega? ¿En eso nos convertimos las diferenciales? También trabajar en función de juntar evidencias, en hacer informes y de justificar la expulsión injusta de estudiantes. Creo que no. Trato de no pensar mucho en eso para no deprimirme, al final igual podemos hacer cosas por los niños que necesitan ayuda a pesar de lo vulnerable y de lo adverso del contexto, de la escuela y lo precario del trabajo (Claudia, $30 \mathrm{de}$ junio de 2018, bitácora).

Claudia, en esta bitácora, evidenció una producción de una identidad docente tensa, confusa y vulnerable ante los mandatos de las políticas de rendición de cuentas y ante las demandas de estandarización/exclusión a las que las interpelaban las autoridades de su institución escolar.

En el caso particular de Claudia, en su discurso dio cuenta de un agobio y un agotamiento a causa de estas demandas contradictorias, que la tensionaban, que la hacían dudar y cuestionar su trabajo pedagógico como profesora encargada de la inclusión escolar. Además, la educadora trataba de no reflexionar demasiado ante estas problemáticas, ya que la llevaban a "deprimirse". A pesar de ello y de toda la adversidad, consideraba que era su deber y responsabilidad preocuparse por la inclusión y el trabajo con los estudiantes con mayores dificultades de aprendizaje y, al mismo tiempo, tratar de resistir y defender su rol a pesar de las múltiples presiones a las que estaba expuesta en su trabajo pedagógico cotidiano. 


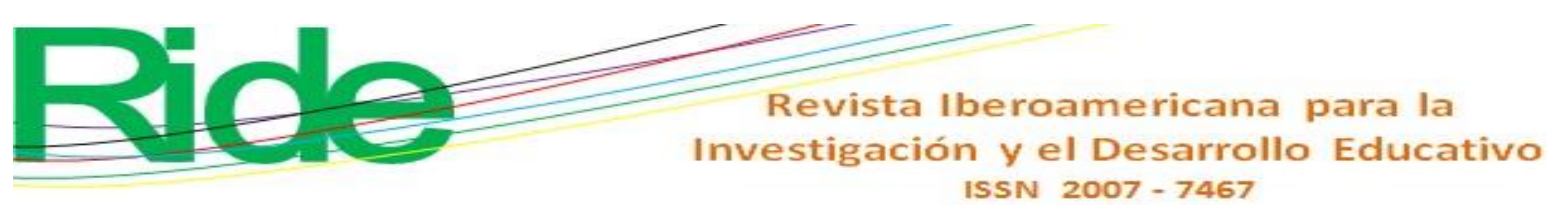

\section{Discusión}

Teniendo en consideración los hallazgos presentados, las resistencias identificadas y materializadas por medio de contraconductas por parte de las educadoras, estas acciones emergieron ante los mandatos de estandarización de la enseñanza para preparar a los alumnos para la evaluación Simce, al igual que como efecto de las prácticas de derivación de educandos con mayores de dificultades de aprendizaje a escuelas de educación especial.

Respecto a la estandarización de la enseñanza, las educadoras desplegaron contraconductas colectivas e individuales. Para el caso de Claudia, se cristalizaron por medio de un acuerdo grupal entre las docentes que logró dos cosas: obedecer en apariencia los mandatos de preparación de alumnos para el Simce y ajustar los ensayos para esta evaluación en función de mejorar los conocimientos y las habilidades más descendidas de los estudiantes en las asignaturas de Lenguaje y Matemáticas.

El hallazgo anterior concuerda parcialmente con investigaciones que se han realizado en el plano nacional con profesores de educación primaria, quienes han resistido a la estandarización de los procesos educativos a través de reclamos a sus directivos o internalizando el malestar que les producía el estar desarrollando un trabajo pedagógico que va en contra de sus identidades y de su ética (Assaél et al., 2014; Rojas y Leyton, 2014). Igualmente, estudios empíricos a nivel internacional han evidenciado resistencia por parte de los maestros, manifestada por medio de murmullos, molestia, ansiedad y estrés ante las demandas de preparación y entrenamiento de alumnos para rendir evaluaciones estandarizadas (Ball, Braun y Maguire, 2011; Braun y Maguire, 2018).

En cuanto al caso de Pamela, esta produjo una resistencia que se desplegó por medio de una contraconducta individual: desobedeció al mandato de aplicar un ensayo para preparar a los estudiantes para el Simce y realizó en su lugar otra actividad pedagógica acorde a su criterio profesional como educadora diferencial encargada de la inclusión; de esta forma omitió la demanda de estandarización de los procesos de enseñanza-aprendizaje. Esta resistencia la pudo ejecutar en un espacio del establecimiento en el que se desempeñaba y que se encontraba alejado de la mirada evaluadora de directivos y supervisores, o del panóptico, desde la analítica foucaultiana, lugar que correspondía al aula de recursos del establecimiento educacional. 


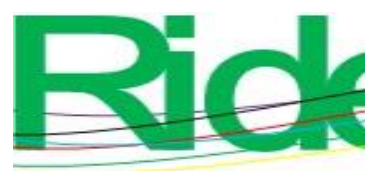

Revista Iberoamericana para la

Investigación y el Desarrollo Educativo

ISSN 2007 - 7467

En lo que respecta a la derivación de educandos a escuelas de educación especial, resultó ser una temática controversial en ambos casos de estudio. Claudia, dado el contexto en el que se encontraba, tensionado ante un eventual cierre de la escuela en caso de no mejorar los resultados en la evaluación Simce, desplegó una contraconducta que la llevó a asociarse con la madre de una alumna que iba a ser derivada a una escuela de educación especial. Esta pedagoga concertó una reunión y acordó con la apoderada un plan pedagógico para mejorar los desempeños de la estudiante en cuestión en Lenguaje y Matemáticas, asignaturas claves en el currículo chileno y en el Simce, acción que ayudaría a impedir la derivación. En tanto que Pamela, en un escenario educativo sin una presión exacerbada, manifestó que prefería seguir su ética como profesional encargada de la inclusión escolar y renunciar a su puesto laboral ante la demanda de derivar a educandos al sistema de educación especial.

En ambos casos, la derivación de alumnos a escuelas de educación especial fue interpretada por las participantes como una práctica que las interpelaba profesional y éticamente, tal y como lo ha estudiado Falabella (2019), es decir, las educadoras se encontraban ante la encrucijada de ser agentes de exclusión de educandos, lo que resultaba ser contrario a su labor como encargadas de la inclusión y, a su vez, se detectó que las participantes se hiperresponsabilizaban de sus alumnos que, además de ser considerados con dificultades de aprendizaje, provenían de sectores de pobreza urbana extrema, por lo que enviarlos a dichas instituciones de educación especial representaba para Claudia y Pamela un daño profundo en las trayectorias educativas y vitales de estos estudiantes.

El resultado anterior concuerda parcialmente con los hallazgos de la investigadora Grinberg (2019), quien propone que, en la época del gerenciamiento, los docentes deben hacerse cargo de la enseñanza y de las vidas de sus educandos en contextos de pobreza abyecta, en donde el Estado ha dejado a su suerte a las escuelas, profesores y niños, y en donde son los mismos docentes quienes, además de luchar por mantener a las escuelas funcionando, se hacen responsables de la calidad de la educación, de la inclusión, del bienestar y de la integralidad de la formación que reciben sus alumnos.

Lo que esta investigación permite iluminar y visibilizar es cómo las políticas de rendición de cuentas en su puesta en acto, tal y como Ball et al. (2011) lo conceptualizan, generan "efectos no deseados" en las escuelas y en las subjetividades de los profesores y niños. En el caso de este estudio, se da cuenta de las diversas contraconductas que las educadoras encargadas de la inclusión tuvieron que desplegar para resistir a la exclusión de 


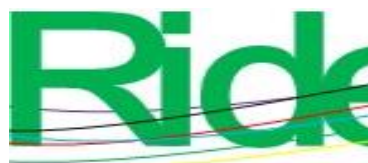

Revista Iberoamericana para la

Investigación y el Desarrollo Educativo ISSN $2007-7467$

los alumnos con dificultades de aprendizaje, tanto para evitar que estos educandos fueran sujetos de la estandarización como también de la eugenesia educativa, término acuñado por Baker (2002), es decir, que fueran enviados a espacios educativos en los que se encierra y se recluye a aquellos sujetos escolares que se escapan a la norma deseada y esperada por el sistema educativo chileno (Infante, Matus y Vizcarra, 2011).

\section{Conclusiones}

La presente investigación da cuenta de las diversas contraconductas que las participantes desplegaron como formas de resistencia ante las lógicas de rendición de cuentas o accountability. Ambas eran interpeladas por los directivos, que también se encontraban tensionados por estandarizar a los educandos, para preparar a estos para la evaluación Simce y para derivar a escuelas de educación especial a aquellos que fueron codificados como "problemáticos" o "riesgosos" debido a sus dificultades de aprendizaje y el eventual bajo puntaje que podrían producir en la prueba ya mencionada.

Ante escuelas y actores que se encuentran tensionados por las lógicas de la rendición de cuentas con altas consecuencias, la agenda política de cada institución se trastoca y pasa a convertirse en una prioridad el "entrenamiento" de los niños para obtener un buen puntaje en la evaluación Simce. En el caso de Claudia, dadas sus condiciones contractuales precarias y de urgencia de su escuela amenazada, esta docente, junto a sus colegas, de manera colectiva, acordaron desplegar contraconductas vinculadas con una "aparente" obediencia en la preparación de alumnos para la evaluación Simce. No obstante, en realidad realizaban un trabajo diferenciado y ajustado a las necesidades y habilidades de los estudiantes con mayores dificultades de aprendizaje. En tanto que Pamela generó una resistencia más explícita e individual, ya que, en el aula de recursos, no les aplicaba los ensayos para el Simce a los educandos con dificultades del aprendizaje.

Respecto a la derivación de alumnos a escuelas de educación especial, esta práctica se había realizado en ambas instituciones educativas y era un tema controvertido. En este sentido, se identificó que se les solicitaba a las educadoras diferenciales evaluar a niños que podrían "perjudicar" a los centros escolares dado el potencial bajo puntaje en la evaluación Simce que podrían producir. En el caso de Claudia, esta desplegó una contraconducta al trabajar con la madre de una alumna que iba ser derivada. Pamela, por su parte, mencionó 


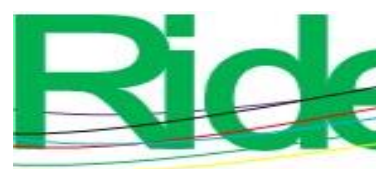

Revista Iberoamericana para la Investigación y el Desarrollo Educativo ISSN $2007-7467$

Pedagógicos 42(2), 395-413. Recuperado de https://dx.doi.org/10.4067/S071807052016000200023.

Fardella, C. y Sisto, V. (2015). Nuevas regulaciones del trabajo docente en Chile: Discurso, subjetividad y resistencia. Psicologia e Sociedade, 27(1), 68-79. Recuperado de https://dx.doi.org/10.1590/1807-03102015v27n1p068.

Foucault, M. (1994). Verdad y Poder. Diálogo con M. Fontana. En Un diálogo sobre el poder y otras conversaciones (pp. 128-145). Barcelona, España: Altaya.

Foucault, M. (2006). Seguridad, territorio y población. Curso en el College de France (19771978). Buenos Aires, Argentina: Siglo XXI Editores.

Foucault, M. (2013). La arqueología del saber. Buenos Aires, Argentina: Siglo XXI Editores.

Grinberg, S. (2019). Self-made school and the everyday making in Buenos Aires slums. British Journal of Sociology of Education, 40(4), 560-577.

Guber, R. (2004). El salvaje metropolitano. Reconstrucción del conocimiento social en el trabajo de campo. Buenos Aires, Argentina: Paidós.

Infante, M., Matus, C. y Vizcarra, R. (2011). Razonando sobre la idea de diferencia en las políticas educativas chilenas. Universum, 26(2), 143-163. Recuperado de http://dx.doi.org/10.4067/S0718-23762011000200008.

Inostroza, F. (2020). La identidad de las educadoras diferenciales en tiempos de políticas de accountability. Archivos Analíticos de Políticas Educativas, 28(29). Recuperado de https://epaa.asu.edu/ojs/article/viewFile/4577/2390.

Larraín, J. (2011). El concepto de ideología 4: Postestructuralismo, postmodernismo y postmarxismo. Santiago, Chile: LOM Ediciones.

Maroy, C. y Voisin, A. (2013). As transformações recentes das políticas de accountability na educação: desafios e incidências das ferramentas de ação pública. Educação \& Sociedade, 34(124), 881-901.

Parcerisa, L. and Falabella, A. (2017). The consolidation of the evaluative state through accountability policies: Trajectory, enactment and tensions in the Chilean education system. Education Policy Analysis Archives, 25(89), 1-27. Retrieved from https://epaa.asu.edu/ojs/article/view/3177.

Raczynski, D., Muñoz, G., Weinstein, J. y Pascual, J. (2016). Subvención Escolar Preferencial (SEP) en Chile: Un Intento por Equilibrar la macro y micro política 


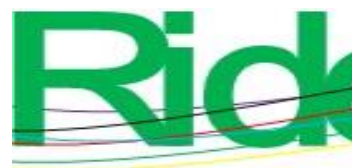

Revista Iberoamericana para la Investigación y el Desarrollo Educativo ISSN 2007 - 7467

escolar. REICE. Revista Iberoamericana Sobre Calidad, Eficacia y Cambio En Educación, 11(2), 165-193. Recuperado de https://revistas.uam.es/index.php/reice/article/view/2902.

Rockwell, E. (2009). La experiencia etnográfica. Historia y cultura en los procesos educativos. Buenos Aires, Argentina: Paidós.

Rojas, M. y Leyton, D. (2014). La nueva subjetividad docente. Construcción de subjetividades docentes en los inicios de la implementación de la Subvención Escolar Preferencial en Chile. Estudios Pedagógicos, 40(1), 205-221. Recuperado de https://dx.doi.org/10.4067/S0718-07052014000200012.

Román, M. y Murillo, J. (2012). Políticas educativas de apoyo a escuelas de sectores pobres y de bajo logro académico en Chile: 1990-2011. Revista de Educación, (número extraordinario), 46-66.

Tyack, D. y Cuban, L. (2001). En busca de la utopía. Un siglo de reformas de las escuelas públicas. Ciudad de México, México: Fondo de Cultura Económica.

Verger, A., Bonal, X. y Zancajo, A. (2016). Recontextualización de políticas y (cuasi) mercados educativos. Un análisis de las dinámicas de demanda y oferta escolar en Chile. Archivos Analíticos de Políticas Educativas, 24(27), 1-27. Recuperado de 10.14507/epaa.24.2098. 